\title{
On New Unified Bounds for a Family of Functions via Fractional $q$ -Calculus Theory
}

\author{
Li Xu, ${ }^{1}$ Yu-Ming Chu $\mathbb{D}^{2,3}$ Saima Rashid ${ }^{1},{ }^{4}$ A. A. El-Deeb $\mathbb{D}^{5},{ }^{5}$ \\ and Kottakkaran Sooppy Nisar ${ }^{6}$ \\ ${ }^{1}$ Jinhua Radio and Television University, Jinhua 321000, China \\ ${ }^{2}$ Department of Mathematics, Huzhou University, Huzhou 313000, China \\ ${ }^{3}$ Hunan Provincial Key Laboratory of Mathematical Modeling and Analysis in Engineering, Changsha University of Science \\ \& Technology, Changsha 410114, China \\ ${ }^{4}$ Department of Mathematics, Government College University, Faisalabad, Pakistan \\ ${ }^{5}$ Department of Mathematics, Faculty of Science, Al-Azhar University, Nasr City, Cairo, Egypt \\ ${ }^{6}$ Department of Mathematics, College of Arts and Sciences, Prince Sattam bin Abdulaziz University, \\ Wadi Al Dawasir 11991, Saudi Arabia
}

Correspondence should be addressed to Yu-Ming Chu; chuyuming@zjhu.edu.cn

Received 11 May 2020; Accepted 25 June 2020; Published 16 July 2020

Academic Editor: Hugo Leiva

Copyright (c) $2020 \mathrm{Li} \mathrm{Xu}$ et al. This is an open access article distributed under the Creative Commons Attribution License, which permits unrestricted use, distribution, and reproduction in any medium, provided the original work is properly cited.

\begin{abstract}
The present article deals with the new estimates in $q$-calculus and fractional $q$-calculus on a time scale $\mathbb{T}_{t_{0}}=\{0\} \cup\left\{t: t=t_{0} q^{n}, n\right.$ is a nonnegative integer $\}$, where $t_{0} \in \mathbb{R}$ and $0<q<1$. The role of fractional time scale $q$-calculus can be found as one of the prominent techniques to generate some variants for a class of positive functions $n(n \in \mathbb{N})$. Finally, our work will provide foundation and motivation for further investigation on time-fractional $q$-calculus systems that have an intriguing application in quantum theory and special relativity theory.
\end{abstract}

\section{Introduction}

Fractional differential equations were executed to demonstrate tremendous innovations for different issues in the physical sciences [1-15]. Since most frameworks involve recollections, the scientists are agreeing with the nonlocality of the fractional operators make it progressively functional in demonstrating the classical derivatives. Recently, nonlocal fractional derivatives without the singular kernel have been exhibited and contemplated $[16,17]$. However, there are no solid numerical defenses of the new sorts of fractional derivatives; their applications were demonstrated by numerous analysts $[18,19]$. Furthermore, presently we have the utilization of fractional calculus in fields like science, material science, and building and among different zones. It is a stunner of the fractional calculus that we have such a large number of valuable meanings of differential and integral operators, for instance, Saigo, conformable, Riemann-Liouville, Katugampola, Hadamard, Erdélyi-Kober, Liouville, local, and Weyl types. These operators are having their significance and applications in picture handling, science, hydrodynamics, and viscoelastic. For a detailed depiction of the origination of fractional calculus, advancement, and applications, we refer the interested readers to the notable books and research articles [20-22].

Hilger [23] began the theories of time scales in his doctoral dissertation and combined discrete and continuous analysis $[24,25]$. At that time, this theory has received a lot of attention. In the book written by Bohner and Peterson [26] on the issues of time scale, a brief summary is given and several time calculations are performed. Over the past decade, many analysts working in special applications have proved a reasonable number of dynamic inequalities on a time scale $[27,28]$. Several researchers have created various 
results relating to fractional calculus on time scales to obtain the corresponding dynamic variants [29].

In the eighteenth century (1707-1783), Euler initiated calculus with no limits refer to as quantum calculus. Jackson began a deliberate investigation of $q$-calculus and presented the $q$-definite integrals. Additionally, he was the first to create $q$-calculus in an efficient manner. Few selected branches of pure and applied mathematics, such as combinatorics, Gauss hypergeometric functions, orthogonal polynomials, dynamic, and quantum theory, have been enhanced by the exploration work of different researchers.

Motivated, by what we mentioned above, we extend the idea of fractional $q$-calculus type operators with a time scale to arbitrary positive order, provide several bounds for a family of $n \in \mathbb{N}$, and finally prove several variants for timefractional $q$-calculus theory. These new results have utilities in the monotonicity for this nabla continuous fractional operator with singular and nonsingular kernel and compare them to the discrete classical ones. The time-fractional $q$-calculus under consideration in this paper have kernels different from classical nabla fractional differences with kernels depending on the rising factorial powers, and we believe that they bring new kernels with new memories, which may be of different interest for applications. The idea is quite new and seems to have opened new doors of investigation towards various scientific fields of research including engineering, fluid dynamics, meteorology, analysis, and aerodynamics.

Inequalities have wild applications in pure and applied mathematics [30-33]. Very recently, many new inequalities such as Hermite-Hadamard type inequality [34-38], Petrović type inequality [39], Pólya-Szegö type inequality [40], Ostrowski type inequality [41], reverse Minkowski inequality [42], Jensen type inequality [43, 44], Bessel function inequality [45], trigonometric and hyperbolic functions inequalities [46], fractional integral inequality [47-51], complete and generalized elliptic integrals inequalities [52-57], generalized convex function inequality [58-60], and mean values inequality [61-63] have been discovered by many researchers.

Variants regarding fractional integral operators are the use of noteworthy significant strategies amongst researchers and accumulate fertile functional applications in various areas of science $[64,65]$. We state some of them, that is, the variants of Minkowski, Hardy, Opial, Hermite-Hadamard, Grüss, Lyenger, Ostrowski, C ebyšev, and Pólya-Szegö, and others. Such applications of fractional integral operators compelled us to show the generalization by using a family of $n$ positive functions involving time-fractional $q$-calculus integrals operators.

Owing to the above phenomena, the key aim of this research is to demonstrate the notations and primary definitions of our noteworthy time-fractional $q$-calculus operator. Also, we present the results concerning for a class of family of $n(n \in \mathbb{N})$ continuous positive decreasing functions on $\left[\varsigma_{1}, \varsigma_{2}\right]$ by employing a time-fractional $q$-calculus operator. Finally, it is emphasized that combining these two approaches, $q$-fractional calculus and time scale analysis, could be the most efficient way of incorporating inequalities into both times and $q$-components for quantum theory and special relativity theory.

\section{Preliminaries}

Let us recall some necessary definitions and preliminary results that are used for further discussion. For more details, we may refer to [33].

Definition 1 (See [33]). The particular time scale $\mathbb{T}_{t_{0}}$ is defined by

$\mathbb{T}_{t_{0}}=\left\{t: t=t_{0} q^{n}, n\right.$ is a nonnegative integer $\} \cup\{0\}, 0<q<1$.

If there is no confusion concerning $t_{0}$, we will denote $\mathbb{T}_{t_{0}}$ by $\mathbb{T}$.

Definition 2. The $q$-factorial function is defined in the following way

$$
\begin{aligned}
& (\zeta-\varphi)^{-(n)}=(\zeta-\varphi)(\zeta-q \varphi)\left(\zeta-q^{\varphi}\right) \cdots\left(\zeta-q^{n} \varphi\right), n \in \mathbb{N}, \\
& (\zeta-\varphi)^{-(n)}=\zeta^{n} \prod_{\kappa=0}^{\infty} \frac{1-(\varphi / \zeta) q^{\kappa}}{1-(\varphi / \zeta) q^{n+\kappa}}, \quad n \notin \mathbb{N} .
\end{aligned}
$$

Definition 3. The $q$-derivative of the $q$-factorial function with respect to $\zeta$ is defined by

$$
\nabla_{q}(\zeta-\varphi)^{-(n)}=\frac{1-q^{n}}{1-q}(\zeta-\varphi)^{\frac{(n-1)}{}}
$$

and the $q$-derivative of the $q$-factorial function with respect to $s$ is defined by

$$
\nabla_{q}(\zeta-\varphi)^{\frac{(n)}{-}}=-\frac{1-q^{n}}{1-q}(\zeta-q \varphi)^{\frac{(n-1)}{-}} .
$$

Definition 4. The $q$-exponential function is defined as

$$
e_{q}(\zeta)=\prod_{\kappa=0}^{\infty}\left(1-q^{\kappa} \zeta\right), \quad e_{q}(0)=1
$$

Definition 5. The $q$-Gamma function is defined by

$$
\Gamma_{q}(\beta)=\frac{1}{1-q} \int_{0}^{1}\left(\frac{\zeta}{1-q}\right)^{\beta-1} e_{q}(q \zeta) \nabla \zeta, \beta \in \mathbb{R}^{+} .
$$

Remark 6. We observe that

$$
\Gamma_{q}(\beta+1)=[\beta]_{q} \Gamma_{q}(\beta), \quad \beta \in \mathbb{R}^{+},
$$

and $[\beta]_{q}=1-q^{\beta} / 1-q$. 
Definition 7. The fractional $q$-integral is defined as

$$
\nabla_{q}^{-\beta} \Psi(\zeta)=\frac{1}{\Gamma_{q}(\beta)} \int_{\zeta_{1}}^{\zeta}(\zeta-q \varphi)^{\frac{\beta-1}{}} \Psi(\varphi) \nabla \varphi
$$

Remark 8. Let $\Psi(\zeta)=1$. Then Definition 7 gives

$$
\nabla_{q}^{-\beta}(1)=\frac{1}{\Gamma_{q}(\beta)} \frac{q-1}{q^{\beta}-1} \zeta^{\underline{\beta}}=\frac{1}{\Gamma_{q}(\beta+1)} \zeta^{\underline{\beta}} .
$$

\section{Main Results}

Now we demonstrate the left fractional $q$ integral operator on an arbitrary time scale $\mathbb{T}$ to derive the generalization of some classical inequalities.

Theorem 9. Let $\alpha>0, \eta \geq \delta>0, \beta \in \mathbb{C}$ with $\Re(\beta)>0$, and $\Psi$ be a continuous positive decreasing function defined on $\mathbb{T}_{t_{0}}$. Then, one has

$$
\frac{\nabla_{\zeta_{1}^{+}, q}^{-\beta}\left[\Psi^{\eta}(\zeta)\right]}{\nabla_{\varsigma_{1}^{+}, q}^{-\beta}\left[\Psi^{\delta}(\zeta)\right]} \geq \frac{\nabla_{\varsigma_{1}^{+}, q}^{-\beta}\left[\left(\zeta-\varsigma_{1}\right)^{\alpha} \Psi^{\eta}(\zeta)\right]}{\nabla_{\varsigma_{1}^{+}, q}^{-\beta}\left[\left(\zeta-\varsigma_{1}\right)^{\alpha} \Psi^{\delta}(\zeta)\right]} .
$$

Proof. Using the hypothesis given in Theorem 9, we have

$$
\left(\left(\omega-\varsigma_{1}\right)^{\alpha}-\left(\varphi-\varsigma_{1}\right)^{\alpha}\right)\left(\Psi^{\eta-\delta}(\varphi)-\Psi^{\eta-\delta}(\omega)\right) \geq 0,
$$

where $\alpha>0, \eta \geq \delta>0$, and $\varphi, \omega \in\left[\varsigma_{1}, \zeta\right]$.

It follows from (11) that

$$
\begin{aligned}
& \left(\omega-\varsigma_{1}\right)^{\alpha} \Psi^{\eta-\delta}(\varphi)-\left(\varphi-\varsigma_{1}\right)^{\alpha} \Psi^{\eta-\delta}(\omega)-\left(\omega-\varsigma_{1}\right)^{\alpha} \Psi^{\eta-\delta}(\omega) \\
& \quad+\left(\varphi-\varsigma_{1}\right)^{\alpha} \Psi^{\eta-\delta}(\varphi) \geq 0 .
\end{aligned}
$$

Multiplying (12) by $1 / \Gamma_{q}(\beta)(\zeta-q \varphi)^{\beta-1 /} \Psi^{\delta}(\varphi), \varphi \in\left(\varsigma_{1}, \zeta\right)$, we have

$$
\begin{aligned}
& \frac{1}{\Gamma_{q}(\beta)}(\zeta-q \varphi)^{\beta-1}\left[\left(\omega-\varsigma_{1}\right)^{\alpha} \Psi^{\eta-\delta}(\varphi)-\left(\varphi-\varsigma_{1}\right)^{\alpha} \Psi^{\eta-\delta}(\omega)\right. \\
& \left.-\left(\omega-\varsigma_{1}\right)^{\alpha} \Psi^{\eta-\delta}(\omega)+\left(\varphi-\varsigma_{1}\right)^{\alpha} \Psi^{\eta-\delta}(\varphi)\right] \Psi^{\delta}(\varphi) \\
& =\left(\omega-\varsigma_{1}\right)^{\alpha} \frac{1}{\Gamma_{q}(\beta)}(\zeta-q \varphi)^{\frac{\beta-1}{-}} \Psi^{\delta}(\varphi) \Psi^{\eta-\delta}(\varphi) \\
& -\left(\varphi-\varsigma_{1}\right)^{\alpha} \frac{1}{\Gamma_{q}(\beta)}(\zeta-q \varphi)^{\beta-1} \Psi^{\delta}(\varphi) \Psi^{\eta-\delta}(\omega) \\
& -\left(\omega-\varsigma_{1}\right)^{\alpha} \frac{1}{\Gamma_{q}(\beta)}(\zeta-q \varphi)^{\frac{\beta-1}{-1}} \Psi^{\delta}(\varphi) \Psi^{\eta-\delta}(\omega) \\
& +\left(\varphi-\varsigma_{1}\right)^{\alpha} \frac{1}{\Gamma_{q}(\beta)}(\zeta-q \varphi)^{\frac{\beta-1}{-}} \Psi^{\delta}(\varphi) \Psi^{\eta-\delta}(\varphi) \geq 0 .
\end{aligned}
$$

Integrating on both sides of (13) for $\varphi$ over $\left(\varsigma_{1}, \zeta\right)$, we have

$$
\begin{gathered}
\left(\omega-\varsigma_{1}\right)^{\alpha} \int_{\varsigma_{1}}^{\zeta} \frac{1}{\Gamma_{q}(\beta)}(\zeta-q \varphi)^{\beta-1} \Psi^{\delta}(\varphi) \Psi^{\eta-\delta}(\varphi) \nabla \varphi-\left(\varphi-\varsigma_{1}\right)^{\alpha} \\
\cdot \int_{\varsigma_{1}}^{\zeta} \frac{1}{\Gamma_{q}(\beta)}(\zeta-q \varphi)^{\beta-1} \Psi^{\delta}(\varphi) \Psi^{\eta-\delta}(\omega) \nabla \varphi-\left(\omega-\varsigma_{1}\right)^{\alpha} \\
\cdot \int_{\varsigma_{1}}^{\zeta} \frac{1}{\Gamma_{q}(\beta)}(\zeta-q \varphi)^{\frac{\beta-1}{-1}} \Psi^{\delta}(\varphi) \Psi^{\eta-\delta}(\omega) \nabla \varphi+\left(\varphi-\varsigma_{1}\right)^{\alpha} \\
\cdot \int_{\varsigma_{1}}^{\zeta} \frac{1}{\Gamma_{q}(\beta)}(\zeta-q \varphi)^{\beta-1} \Psi^{\delta}(\varphi) \Psi^{\eta-\delta}(\varphi) \nabla \varphi \geq 0,
\end{gathered}
$$

that is

$$
\begin{aligned}
& \left(\omega-\varsigma_{1}\right)^{\alpha}\left(\nabla_{\zeta_{1}^{+}, q}^{-\beta}\left[\Psi^{\eta}(\zeta)\right]\right)+\Psi^{\eta-\delta}(\omega)\left(\nabla_{\mathcal{C}^{+}, q}^{-\beta}\left[\left(\zeta-\varsigma_{1}\right)^{\alpha} \Psi^{\delta}(\zeta)\right]\right) \\
& \quad-\left(\omega-\varsigma_{1}\right)^{\alpha} \Psi^{\eta-\delta}(\omega)\left(\nabla_{\zeta_{1}^{+}, q}^{-\beta}\left[\Psi^{\delta}(\zeta)\right]\right)-\left(\left[\left(\zeta-\varsigma_{1}\right)^{\alpha} \Psi^{\eta}(\zeta)\right]\right) \geq 0
\end{aligned}
$$

Multiplying (15) by $1 / \Gamma_{q}(\beta)(\zeta-q \omega)^{\beta-1 /} \Psi^{\delta}(\omega), \omega \in\left(\varsigma_{1}\right.$, $\zeta)$, and integrating for $\omega$ over $\left(\varsigma_{1}, \zeta\right)$ shows

$$
\begin{aligned}
& \left(\nabla_{\mathcal{C}_{1}^{+}, q}^{-\beta}\left[\Psi^{\eta}(\zeta)\right]\right)\left(\nabla_{\varsigma_{1}^{+}, q}^{-\beta}\left[\left(\zeta-\varsigma_{1}\right)^{\alpha} \Psi^{\delta}(\zeta)\right]\right) \\
& \quad-\left(\nabla_{\varsigma_{1}^{+}, q}^{-\beta}\left[\left(\zeta-\varsigma_{1}\right)^{\alpha} \Psi^{\eta}(\zeta)\right]\right)\left(\left[\Psi^{\delta}(\zeta)\right]\right) \geq 0 .
\end{aligned}
$$

Dividing the above inequality by $\left(\nabla_{\varsigma_{1}^{+}, q}^{-\beta}\left[\left(\zeta-\varsigma_{1}\right)^{\alpha} \Psi^{\delta}(\zeta)\right]\right)$ $\left(\nabla_{\mathcal{S}_{1}^{+}, q}^{-\beta}\left[\Psi^{\delta}(\zeta)\right]\right)$, we get the desired inequality (10).

Theorem 10. Let $\alpha>0$, and $\eta \geq \delta>0, \beta, \lambda \in \mathbb{C}$ with $\mathfrak{R}(\beta)$ $>0$ and $R(\lambda)>0$, and $\Psi$ be a continuous positive decreasing function defined on $\mathbb{T}_{t_{0}}$. Then the time-fractional q-integral satisfies the inequality

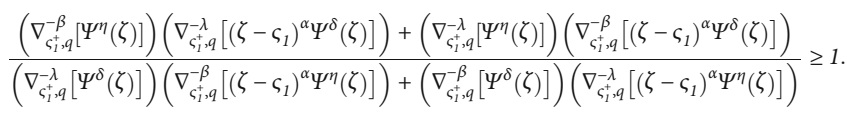

Proof. Multiplying both sides of (15) by $1 / \Gamma_{q}(\lambda)(\zeta-q \omega)^{\lambda-1 /}$ $\Psi^{\delta}(\omega), \omega \in\left(\varsigma_{1}, \zeta\right)$ and integrating for $\omega$ over $\left(\varsigma_{1}, \zeta\right)$ shows

$$
\begin{aligned}
& \left.\left(\nabla_{\mathcal{C}_{1}^{+}, q}^{-\beta}\left[\Psi^{\eta}(\zeta)\right]\right)\left(\nabla_{\mathcal{C}_{1}^{+}, q}^{-\lambda}\left[\left(\zeta-\varsigma_{1}\right)^{\alpha} \Psi^{\delta}(\zeta)\right]\right)+\nabla_{\mathcal{C}_{1}^{+}, q}^{-\lambda}\left[\Psi^{\eta}(\zeta)\right]\right) \\
& \cdot\left(\nabla_{\mathcal{C}_{1}^{+}, q}^{-\beta}\left[\left(\zeta-\varsigma_{1}\right)^{\alpha} \Psi^{\delta}(\zeta)\right]\right)-\left(\nabla_{\mathcal{C}_{1}^{+}, q}^{-\lambda}\left[\Psi^{\delta}(\zeta)\right]\right) \\
& \cdot\left(\nabla_{\mathcal{C}_{1}^{+}, q}^{-\beta}\left[\left(\zeta-\varsigma_{1}\right)^{\alpha} \Psi^{\eta}(\zeta)\right]\right)-\left(\nabla_{\mathcal{C}_{1}^{+}, q}^{-\beta}\left[\Psi^{\delta}(\zeta)\right]\right) \\
& \cdot\left(\nabla_{\mathcal{C}_{1}^{+}, q}^{-\lambda}\left[\left(\zeta-\varsigma_{1}\right)^{\alpha} \Psi^{\eta}(\zeta)\right]\right) \geq 0 .
\end{aligned}
$$


Dividing (18) by

$$
\begin{aligned}
& \left(\nabla_{\varsigma_{1}^{+}, q}^{-\lambda}\left[\Psi^{\delta}(\zeta)\right]\right)\left(\nabla_{\varsigma_{1}^{+}, q}^{-\beta}\left[\left(\zeta-\varsigma_{1}\right)^{\alpha} \Psi^{\eta}(\zeta)\right]\right) \\
& \quad-\left(\nabla_{\varsigma_{1}^{+}, q}^{-\beta}\left[\Psi^{\delta}(\zeta)\right]\right)\left(\nabla_{\zeta_{1}^{+}, q}^{-\lambda}\left[\left(\zeta-\varsigma_{1}\right)^{\alpha} \Psi^{\eta}(\zeta)\right]\right),
\end{aligned}
$$

we get the desired inequality (17).

Theorem 11. Let $\alpha>0, \eta \geq \delta>0, \beta \in \mathbb{C}$ with $\mathfrak{R}(\beta)>0$, $\Psi$ be a continuous positive decreasing function defined on $\mathbb{T}_{t_{0}}$, and $\hbar$ be a continuous positive increasing function on $\mathbb{T}_{t_{0}}$. Then the time-fractional $q$-integral satisfies the inequality

$$
\frac{\left(\nabla_{\varsigma_{1}^{+}, q}^{-\beta}\left[\Psi^{\eta}(\zeta)\right]\right)\left(\nabla_{\varsigma_{1}^{+}, q}^{-\beta}\left[\Psi^{\delta}(\zeta) \hbar^{\alpha}(\zeta)\right]\right)}{\left(\nabla_{\varsigma_{1}^{+}, q}^{-\beta}\left[\Psi^{\delta}(\zeta)\right]\right)\left(\nabla_{\varsigma_{1}^{+}, q}^{-\beta}\left[\Psi^{\eta}(\zeta) \hbar^{\alpha}(\zeta)\right]\right)} \geq 1 .
$$

Proof. Using the hypothesis given in Theorem 11, we have

$$
\left(\hbar^{\alpha}(\omega)-\hbar^{\alpha}(\varphi)\right)\left(\Psi^{\eta-\delta}(\varphi)-\Psi^{\eta-\delta}(\omega)\right) \geq 0
$$

where $\alpha>0, \eta \geq \delta>0$, and $\varphi, \omega \in\left[\varsigma_{1}, \zeta\right]$. From (21), we have

$$
\begin{aligned}
& \hbar^{\alpha}(\omega) \Psi^{\eta-\delta}(\varphi)-\hbar^{\alpha}(\varphi) \Psi^{\eta-\delta}(\omega) \\
& \quad+\hbar^{\alpha}(\omega) \Psi^{\eta-\delta}(\omega)-\hbar^{\alpha}(\varphi) \Psi^{\eta-\delta}(\varphi) \geq 0 .
\end{aligned}
$$

Taking product of (22) by $1 / \Gamma_{q}(\beta)(\zeta-q \varphi)^{\beta-1 /} \Psi^{\delta}(\varphi), \varphi$ $\in\left(\varsigma_{1}, \zeta\right)$, we get

$$
\begin{aligned}
& \frac{1}{\Gamma_{q}(\beta)}(\zeta-q \varphi)^{\frac{\beta-1}{}} \Psi^{\delta}(\varphi)\left[\hbar^{\alpha}(\omega) \Psi^{\eta-\delta}(\varphi)-\hbar^{\alpha}(\varphi) \Psi^{\eta-\delta}(\omega)\right. \\
& \left.\quad+\hbar^{\alpha}(\omega) \Psi^{\eta-\delta}(\omega)-\hbar^{\alpha}(\varphi) \Psi^{\eta-\delta}(\varphi)\right] \\
& \quad=\hbar^{\alpha}(\omega) \frac{1}{\Gamma_{q}(\beta)}(\zeta-q \varphi)^{\frac{\beta-1}{4}} \Psi^{\eta}(\varphi)-\hbar^{\alpha}(\varphi) \frac{1}{\Gamma_{q}(\beta)} \\
& \quad \cdot(\zeta-q \varphi)^{\frac{\beta-1}{2}} \Psi^{\eta-\delta}(\omega) \Psi^{\delta}(\varphi)+\hbar^{\alpha}(\omega) \frac{1}{\Gamma_{q}(\beta)}(\zeta-q \varphi)^{\frac{\beta-1}{1}} \Psi^{\eta-\delta} \\
& \quad \cdot(\omega) \Psi^{\delta}(\varphi)-\hbar^{\alpha}(\varphi) \frac{1}{\Gamma_{q}(\beta)}(\zeta-q \varphi)^{\frac{\beta-1}{-}} \Psi^{\eta}(\varphi) \geq 0 .
\end{aligned}
$$

Integrating (23) for $\varphi$ over $\left(\varsigma_{1}, \zeta\right)$, we obtain

$$
\begin{aligned}
& \frac{\hbar^{\alpha}(\omega)}{\Gamma_{q}(\beta)} \int_{\zeta_{1}}^{\zeta}(\zeta-q \varphi)^{\frac{\beta-1}{2}} \Psi^{\eta}(\varphi) \nabla \varphi-\frac{\hbar^{\alpha}(\varphi)}{\Gamma_{q}(\beta)} \int_{\zeta_{1}}^{\zeta}(\zeta-q \varphi)^{\frac{\beta-1}{1}} \Psi^{\eta-\delta} \\
& \cdot(\omega) \Psi^{\delta}(\varphi) \nabla \varphi+\frac{\hbar^{\alpha}(\omega)}{\Gamma_{q}(\beta)} \int_{\zeta_{1}}^{\zeta}(\zeta-q \varphi)^{\frac{\beta-1}{}} \Psi^{\eta-\delta}(\omega) \Psi^{\delta}(\varphi) \nabla \varphi \\
& -\frac{\hbar^{\alpha}(\varphi)}{\Gamma_{q}(\beta)} \int_{\varsigma_{1}}^{\zeta}(\zeta-q \varphi)^{\frac{\beta-1}{2}} \Psi^{\eta}(\varphi) \nabla \varphi \geq 0 .
\end{aligned}
$$

It follows that

$$
\begin{aligned}
& \hbar^{\alpha}(\omega)\left(\nabla_{\varsigma_{1}^{+}, q}^{-\beta}\left[\Psi^{\eta}(\zeta)\right]\right)+\Psi^{\eta-\delta}(\omega)\left(\nabla_{\mathcal{\zeta}_{1}^{+}, q}^{-\beta}\left[\hbar^{\alpha}(\zeta) \Psi^{\delta}(\zeta)\right]\right) \\
& \quad-\hbar^{\alpha}(\omega) \Psi^{\eta-\delta}(\omega)\left(\nabla_{\mathcal{\zeta}_{1}^{+}, q}^{-\beta}\left[\Psi^{\delta}(\zeta)\right]\right)-\left(\nabla_{\zeta_{1}^{+}, q}^{-\beta}\left[\hbar^{\alpha}(\zeta) \Psi^{\delta}(\zeta)\right]\right) \geq 0 .
\end{aligned}
$$

Again, taking the product (15) by $1 / \Gamma_{q}(\beta)(\zeta-q \omega)^{\beta-1 /}$ $\Psi^{\delta}(\omega), \omega \in\left(\varsigma_{1}, \zeta\right)$, and integrating for $\omega$ over $\left(\varsigma_{1}, \zeta\right)$ gives

$$
\begin{aligned}
& \left(\nabla_{\varsigma_{1}^{+}, q}^{-\beta}\left[\Psi^{\eta}(\zeta)\right]\right)\left(\nabla_{\varsigma_{1}^{+}, q}^{-\beta}\left[\Psi^{\delta}(\zeta) \hbar^{\alpha}(\zeta)\right]\right) \\
& \quad-\left(\nabla_{\zeta_{1}^{+}, q}^{-\beta}\left[\Psi^{\delta}(\zeta)\right]\right)\left(\nabla_{\zeta_{1}^{+}, q}^{-\beta}\left[\Psi^{\eta}(\zeta) \hbar^{\alpha}(\zeta)\right]\right) \geq 0,
\end{aligned}
$$

which completes the proof of the desired inequality (20).

Theorem 12. Let $\alpha>0, \eta \geq \delta>0, \mathfrak{R}(\lambda), \mathfrak{R}(\beta)>0$ with $\mathfrak{R}$ $(\lambda), \mathfrak{R}(\beta)>0, \Psi$ be a continuous positive decreasing function defined on $\mathbb{T}_{t_{0}}$ and $\hbar$ be a continuous positive increasing function on $\mathbb{T}_{t_{0}}$. Then, one has

$\frac{\left(\nabla_{\varsigma_{1}^{+}, q}^{-\beta}\left[\Psi^{\eta}(\zeta)\right] \nabla_{\varsigma_{1}^{+}, q}^{-\lambda}\left[\hbar^{\alpha}(\zeta) \Psi^{\delta}(\zeta)\right]\right)+\left(\nabla_{\mathcal{C}_{1}^{+}, q}^{-\lambda}\left[\Psi^{\eta}(\zeta)\right] \nabla_{\zeta_{1}^{+}, q}^{-\beta}\left[\hbar^{\theta}(\zeta) \Psi^{\delta}(\zeta)\right]\right)}{\left(\nabla_{\mathcal{C}_{1}^{+}, q}^{-\beta}\left[\hbar^{\alpha}(\zeta) \Psi^{\eta}(\zeta)\right] \nabla_{\varsigma_{1}^{+}, q}^{-\lambda}\left[\Psi^{\delta}(\zeta)\right]\right)+\left(\nabla_{\varsigma_{1}^{+}, q}^{-\lambda}\left[\hbar^{\alpha}(\zeta) \Psi^{\eta}(\zeta)\right] \nabla_{\mathcal{C}_{1}^{+}, q}^{-\lambda}\left[\Psi^{\delta}(\zeta)\right]\right)} \geq 1$.

Proof. Multiplying both sides of $(25)$ by $1 / \Gamma_{q}(\beta)(\zeta-q \omega)^{\beta-1 /}$ $\Psi^{\delta}(\omega), \omega \in\left(\varsigma_{1}, \zeta\right)$, and integrating for $\omega$ over $\left(\varsigma_{1}, \zeta\right)$ leads to the conclusion that

$$
\begin{aligned}
& \left(\nabla_{\varsigma_{1}^{+}, q}^{-\beta}\left[\Psi^{\eta}(\zeta)\right] \nabla_{\varsigma_{1}^{+}, q}^{-\lambda}\left[\hbar^{\alpha}(\zeta) \Psi^{\delta}(\zeta)\right]\right) \\
& \quad+\left(\nabla_{\varsigma_{1}^{+}, q}^{-\lambda}\left[\Psi^{\eta}(\zeta)\right] \nabla_{\varsigma_{1}^{+}, q}^{-\beta}\left[\hbar^{\theta}(\zeta) \Psi^{\delta}(\zeta)\right]\right) \\
& \quad-\left(\nabla_{\varsigma_{1}^{+}, q}^{-\beta}\left[\hbar^{\alpha}(\zeta) \Psi^{\eta}(\zeta)\right] \nabla_{\varsigma_{1}^{+}, q}^{-\lambda}\left[\Psi^{\delta}(\zeta)\right]\right) \\
& -\left(\nabla_{\varsigma_{1}^{+}, q}^{-\lambda}\left[\hbar^{\alpha}(\zeta) \Psi^{\eta}(\zeta)\right] \nabla_{\varsigma_{1}^{+}, q}^{-\lambda}\left[\Psi^{\delta}(\zeta)\right]\right) \geq 0 .
\end{aligned}
$$

It follows that

$$
\begin{aligned}
& \left(\nabla_{\varsigma_{1}^{+}, q}^{-\beta}\left[\Psi^{\eta}(\zeta)\right] \nabla_{\varsigma_{1}^{+}, q}^{-\lambda}\left[\hbar^{\alpha}(\zeta) \Psi^{\delta}(\zeta)\right]\right) \\
& \quad+\left(\nabla_{\mathcal{C}_{1}^{+}, q}^{-\lambda}\left[\Psi^{\eta}(\zeta)\right] \nabla_{\varsigma_{1}^{+}, q}^{-\beta}\left[\hbar^{\theta}(\zeta) \Psi^{\delta}(\zeta)\right]\right) \\
& \quad \geq\left(\nabla_{\varsigma_{1}^{+}, q}^{-\beta}\left[\hbar^{\alpha}(\zeta) \Psi^{\eta}(\zeta)\right] \nabla_{\varsigma_{1}^{+}, q}^{-\lambda}\left[\Psi^{\delta}(\zeta)\right]\right) \\
& \quad+\left(\nabla_{\varsigma_{1}^{+}, q}^{-\lambda}\left[\hbar^{\alpha}(\zeta) \Psi^{\eta}(\zeta)\right] \nabla_{\varsigma_{1}^{+}, q}^{-\lambda}\left[\Psi^{\delta}(\zeta)\right]\right) .
\end{aligned}
$$

Dividing above inequality by

$\left(\nabla_{\mathcal{C}_{1}^{+}, q}^{-\beta}\left[\hbar^{\alpha}(\zeta) \Psi^{\eta}(\zeta)\right] \nabla_{\varsigma_{1}^{+}, q}^{-\lambda}\left[\Psi^{\delta}(\zeta)\right]\right)+\left(\nabla_{C_{1}^{+}, q}^{-\lambda}\left[\hbar^{\alpha}(\zeta) \Psi^{\eta}(\zeta)\right] \nabla_{\varsigma_{1}^{+}, q}^{-\lambda}\left[\Psi^{\delta}(\zeta)\right]\right)$,

we get the desired inequality (27). 
Now, we demonstrate the fractional $q$-integral to derive some inequalities for a class of $n$-decreasing positive functions.

Theorem 13. Let $\alpha>0, \eta \geq \delta_{\kappa}>0$ for any fixed $\kappa \in\{1,2,3$, $\cdots, n\}, \beta \in \mathbb{C}$ with $\mathfrak{R}(\beta)>0$, and $\left\{\Psi_{j}, j=1,2,3, \cdots, n\right\}$ be a sequence of continuous positive decreasing functions defined on $\mathbb{T}_{t_{0}}$. Then, the time-fractional $q$-integral satisfies the inequality

$$
\frac{\nabla_{\mathcal{C}_{1}^{+}, q}^{-\beta}\left[\prod_{j \neq \kappa}^{n} \Psi_{j}^{\delta j} \Psi_{\kappa}^{\eta}(\zeta)\right]}{\nabla_{\varsigma_{1}^{+}, q}^{-\beta}\left[\prod_{j=1}^{n} \Psi_{j}^{\delta j}(\zeta)\right]} \geq \frac{\nabla_{\zeta_{1}^{+}, q}^{-\beta}\left[\left(\zeta-\varsigma_{1}\right)^{\alpha} \prod_{j \neq \kappa}^{n} \Psi_{j}^{\delta j} \Psi_{\kappa}^{\eta}(\zeta)\right]}{\nabla_{\mathcal{C}_{1}^{+}, q}^{-\beta}\left[\left(\zeta-\varsigma_{1}\right)^{\alpha} \prod_{j=1}^{n} \Psi_{j}^{\delta j}(\zeta)\right]} .
$$

Proof. Since $\left\{\Psi_{j}, j=1,2,3, \cdots, n\right\}$ is a sequence of continuous positive decreasing functions on $\left[\varsigma_{1}, \zeta\right]$, we have

$$
\left(\left(\omega-\varsigma_{1}\right)^{\alpha}-\left(\varphi-\varsigma_{1}\right)^{\alpha}\right)\left(\Psi_{\kappa}^{\eta-\delta \kappa}(\varphi)-\Psi_{\kappa}^{\eta-\delta \kappa}(\omega)\right) \geq 0,
$$

for any fixed $\kappa \in\{1,2,3, \cdots, n\}, \alpha>0, \eta \geq \delta_{\kappa}>0$ and $\varphi, \omega \in$ $\left[\varsigma_{1}, \zeta\right]$.

It follows from (32) that

$$
\begin{aligned}
& \left(\omega-\varsigma_{1}\right)^{\alpha} \Psi_{\kappa}^{\eta-\delta \kappa}(\varphi)+\left(\varphi-\varsigma_{1}\right)^{\alpha} \Psi_{\kappa}^{\eta-\delta \kappa}(\omega) \\
& \quad \geq\left(\omega-\varsigma_{1}\right)^{\alpha} \Psi_{\kappa}^{\eta-\delta \kappa}(\omega)+\left(\varphi-\varsigma_{1}\right)^{\alpha} \Psi_{\kappa}^{\eta-\delta \kappa}(\varphi) .
\end{aligned}
$$

Taking the product of (22) by $1 / \Gamma_{q}(\beta)(\zeta-q \varphi)^{\beta-1 /} \prod_{j=1}^{n}$ $\Psi_{j}^{\delta_{j}}(\varphi), \varphi \in\left(\varsigma_{1}, \zeta\right)$, and integrating for $\varphi$ over $\left(\varsigma_{1}, \zeta\right)$, we have

$$
\begin{gathered}
\frac{1}{\Gamma_{q}(\beta)}(\zeta-q \varphi)^{-\beta-1}\left[\left(\omega-\varsigma_{1}\right)^{\alpha} \Psi_{\kappa}^{\eta-\delta \kappa}(\varphi)+\left(\varphi-\varsigma_{1}\right)^{\alpha} \Psi_{\kappa}^{\eta-\delta \kappa}(\omega)\right. \\
\left.-\left(\omega-\varsigma_{1}\right)^{\alpha} \Psi_{\kappa}^{\eta-\delta \kappa}(\omega)-\left(\varphi-\varsigma_{1}\right)^{\alpha} \Psi_{\kappa}^{\eta-\delta \kappa}(\varphi)\right] \prod_{j=1}^{n} \Psi_{j}^{\delta_{j}}(\varphi) \\
=\left(\omega-\varsigma_{1}\right)^{\alpha} \frac{1}{\Gamma_{q}(\beta)}(\zeta-q \varphi)^{\beta-1} \prod_{j=1}^{n} \Psi_{j}^{\delta_{j}}(\varphi) \Psi_{\kappa}^{\eta-\delta \kappa}(\varphi) \\
+\left(\varphi-\varsigma_{1}\right)^{\alpha} \frac{1}{\Gamma_{q}(\beta)}(\zeta-q \varphi)^{\beta-1} \prod_{j=1}^{n} \Psi_{j}^{\delta_{j}}(\varphi) \Psi_{\kappa}^{\eta-\delta \kappa}(\omega) \\
-\left(\omega-\varsigma_{1}\right)^{\alpha} \frac{1}{\Gamma_{q}(\beta)}(\zeta-q \varphi)^{\frac{\beta-1}{n}} \prod_{j=1}^{n} \Psi_{j}^{\delta_{j}}(\varphi) \Psi_{\kappa}^{\eta-\delta \kappa}(\omega) \\
-\left(\varphi-\varsigma_{1}\right)^{\alpha} \frac{1}{\Gamma_{q}(\beta)}(\zeta-q \varphi)^{\frac{\beta-1}{-}} \prod_{j=1}^{n} \Psi_{j}^{\delta_{j}}(\varphi) \Psi_{\kappa}^{\eta-\delta \kappa}(\varphi) \geq 0 .
\end{gathered}
$$

Integrating (34) for $\varphi$ over $\left(\varsigma_{1}, \zeta\right)$, we get

$$
\begin{aligned}
& \left(\omega-\varsigma_{1}\right)^{\alpha} \frac{1}{\Gamma_{q}(\beta)} \int_{\zeta_{1}}^{\zeta}(\zeta-q \varphi)^{-\beta-1} \prod_{j=1}^{n} \Psi_{j}^{\delta_{j}}(\varphi) \Psi_{\kappa}^{\eta-\delta \kappa}(\varphi) \nabla \varphi \\
& +\left(\varphi-\varsigma_{1}\right)^{\alpha} \frac{1}{\Gamma_{q}(\beta)} \int_{\varsigma_{1}}^{\zeta}(\zeta-q \varphi)^{\beta-1} \prod_{j=1}^{n} \Psi_{j}^{\delta_{j}}(\varphi) \Psi_{\kappa}^{\eta-\delta \kappa}(\omega) \nabla \varphi \\
& -\left(\omega-\varsigma_{1}\right)^{\alpha} \frac{1}{\Gamma_{q}(\beta)} \int_{\varsigma_{1}}^{\zeta}(\zeta-q \varphi)^{-\beta-1} \prod_{j=1}^{n} \Psi_{j}^{\delta_{j}}(\varphi) \Psi_{\kappa}^{\eta-\delta \kappa}(\omega) \nabla \varphi \\
& -\left(\varphi-\varsigma_{1}\right)^{\alpha} \frac{1}{\Gamma_{q}(\beta)} \int_{\varsigma_{1}}^{\zeta}(\zeta-q \varphi)^{-\frac{\beta-1}{n}} \prod_{j=1}^{n} \Psi_{j}^{\delta_{j}}(\varphi) \Psi_{\kappa}^{\eta-\delta \kappa}(\varphi) \nabla \varphi \geq 0 .
\end{aligned}
$$

It follows from (35) that

$$
\begin{aligned}
& \left(\omega-\varsigma_{1}\right)^{\alpha} \nabla_{\zeta_{1}^{+}, q}^{-\beta}\left[\prod_{j \neq \kappa}^{n} \Psi_{j}^{\delta j} \Psi_{\kappa}^{\eta}(\zeta)\right]+\Psi_{\kappa}^{\eta-\delta \kappa}(\omega) \nabla_{\mathcal{C}_{1}^{+}, q}^{-\beta}\left[\left(\zeta-\varsigma_{1}\right)^{\alpha} \prod_{j=1}^{n} \Psi_{j}^{\delta j}(\zeta)\right] \\
& \geq\left(\omega-\varsigma_{1}\right)^{\alpha} \Psi_{\kappa}^{\eta-\delta \kappa}(\omega) \nabla_{\zeta_{1}^{1}, q}^{-\beta}\left[\prod_{j=1}^{n} \Psi_{j}^{\delta j}(\zeta)\right] \\
& +\nabla_{\zeta_{1}^{+}, q}^{-\beta}\left[\left(\zeta-\varsigma_{1}\right)^{\alpha} \prod_{j \neq \kappa}^{n} \Psi_{j}^{\delta j} \Psi_{\kappa}^{\eta}(\zeta)\right] .
\end{aligned}
$$

Again, taking the product of (36) by $1 / \Gamma_{q}(\beta)$ $(\zeta-q \omega)^{\beta-1 /} \prod_{j=1}^{n} \Psi_{j}^{\delta_{j}}(\omega), \omega \in\left(\varsigma_{1}, \zeta\right)$, and integrating for $\omega$ over $\left(\varsigma_{1}, \zeta\right)$, we obtain

$$
\begin{aligned}
& \nabla_{\mathcal{C}_{1}^{+}, q}^{-\beta}\left[\prod_{j \neq \kappa}^{n} \Psi_{j}^{\delta j} \Psi_{\kappa}^{\eta}(\zeta)\right] \nabla_{\mathcal{C}_{1}^{+}, q}^{-\beta}\left[\left(\zeta-\varsigma_{1}\right)^{\alpha} \prod_{j=1}^{n} \Psi_{j}^{\delta j}(\zeta)\right] \\
& \geq \nabla_{\zeta_{1}^{+}, q}^{-\beta}\left[\left(\zeta-\varsigma_{1}\right)^{\alpha} \prod_{j \neq \kappa}^{n} \Psi_{j}^{\delta j} \Psi_{\kappa}^{\eta}(\zeta)\right] \nabla_{\mathcal{C}_{1}^{+}, q}^{-\beta}\left[\prod_{j=1}^{n} \Psi_{j}^{\delta j}(\zeta)\right],
\end{aligned}
$$

which gives the desired inequality (31).

Theorem 14. Let $\alpha>0, \eta \geq \delta_{\kappa}>0$ for any fixed $\kappa \in\{1,2,3$, $\cdots, n\}, \beta, \lambda \in \mathbb{C}$ with $\mathfrak{R}(\beta)>0, \Re(\lambda)>0$, and $\left\{\Psi_{j}, j=1,2,3\right.$, $\cdots, n\}$ be a sequence of continuous positive decreasing functions defined on $\mathbb{T}_{t_{0}}$. Then, we have the inequality

$$
\begin{aligned}
& \left(\nabla_{\mathcal{C}_{1}^{\prime}, q}^{-\beta}\left[\prod_{j \neq \kappa}^{n} \Psi_{j}^{\delta j} \Psi_{\kappa}^{\eta}(\zeta)\right] \nabla_{\zeta_{1}^{+}, q}^{-\lambda}\left[\left(\zeta-\varsigma_{1}\right)^{\alpha} \prod_{j=1}^{n} \Psi_{j}^{\delta j}(\zeta)\right]\right. \\
& \left.+\nabla_{\zeta_{1}^{\prime}, q}^{-\lambda}\left[\prod_{j \neq \kappa}^{n} \Psi_{j}^{\delta j} \Psi_{\kappa}^{\eta}(\zeta)\right] \nabla_{\zeta_{1}^{+}, q}^{-\beta}\left[\left(\zeta-\varsigma_{1}\right)^{\alpha} \prod_{j=1}^{n} \Psi_{j}^{\delta j}(\zeta)\right]\right) / \\
& \left(\nabla_{\mathcal{C}_{1}, q}^{-\beta}\left[\left(\zeta-\varsigma_{1}\right)^{\alpha} \prod_{j \neq \kappa}^{n} \Psi_{j}^{\delta j} \Psi_{\kappa}^{\eta}(\zeta)\right] \nabla_{\zeta_{1}^{+}, q}^{-\lambda}\left[\prod_{j=1}^{n} \Psi_{j}^{\delta j}(\zeta)\right]\right. \\
& \left.+\nabla_{\zeta_{1}^{1}, q}^{-\lambda}\left[\left(\zeta-\varsigma_{1}\right)^{\alpha} \prod_{j \neq \kappa}^{n} \Psi_{j}^{\delta j} \Psi_{\kappa}^{\eta}(\zeta)\right] \nabla_{\zeta_{1}^{\prime}, q}^{-\beta}\left[\prod_{j=1}^{n} \Psi_{j}^{\delta j}(\zeta)\right]\right) \geq 1 .
\end{aligned}
$$


Proof. Taking product on both sides of (36) by $1 / \Gamma_{q}(\lambda)$ $(\zeta-q \theta)^{\lambda-1 /} \prod_{j=1}^{n} \Psi_{j}^{\delta_{j}}(\omega), \omega \in\left(\varsigma_{1}, \zeta\right)$, and integrating for $\omega$ over $\left(\varsigma_{1}, \zeta\right)$, we get

$$
\begin{aligned}
& \nabla_{\zeta_{1}^{1}, q}^{-\beta}\left[\prod_{j \neq \kappa}^{n} \Psi_{j}^{\delta j} \Psi_{\kappa}^{\eta}(\zeta)\right] \nabla_{\zeta_{1}^{1}, q}^{-\lambda}\left[\left(\zeta-\zeta_{1}\right)^{\alpha} \prod_{j=1}^{n} \Psi_{j}^{\delta j}(\zeta)\right] \\
& +\nabla_{\zeta_{1}^{+}, q}^{-\lambda}\left[\prod_{j \neq \kappa}^{n} \Psi_{j}^{\delta j} \Psi_{\kappa}^{\eta}(\zeta)\right] \nabla_{\zeta_{1}^{+}, q}^{-\beta}\left[\left(\zeta-\varsigma_{1}\right)^{\alpha} \prod_{j=1}^{n} \Psi_{j}^{\delta j}(\zeta)\right] \\
& \geq \nabla_{\zeta_{1}^{+}, q}^{-\beta}\left[\left(\zeta-\varsigma_{1}\right)^{\alpha} \prod_{j \neq \kappa}^{n} \Psi_{j}^{\delta j} \Psi_{\kappa}^{\eta}(\zeta)\right] \nabla_{\zeta_{1}^{+}, q}^{-\lambda}\left[\prod_{j=1}^{n} \Psi_{j}^{\delta j}(\zeta)\right] \\
& +\nabla_{\zeta_{1}^{+}, q}^{-\lambda}\left[\left(\zeta-\varsigma_{1}\right)^{\alpha} \prod_{j \neq \kappa}^{n} \Psi_{j}^{\delta j} \Psi_{\kappa}^{\eta}(\zeta)\right] \nabla_{\mathcal{C}_{1}^{+}, q}^{-\beta}\left[\prod_{j=1}^{n} \Psi_{j}^{\delta j}(\zeta)\right] .
\end{aligned}
$$

Dividing the above inequality by

$$
\begin{aligned}
& \nabla_{\zeta_{1}^{+}, q}^{-\beta}\left[\left(\zeta-\zeta_{1}\right)^{\alpha} \prod_{j \neq \kappa}^{n} \Psi_{j}^{\delta j} \Psi_{\kappa}^{\eta}(\zeta)\right] \nabla_{\zeta_{1}^{+}, q}^{-\lambda}\left[\prod_{j=1}^{n} \Psi_{j}^{\delta j}(\zeta)\right] \\
& +\nabla_{\zeta_{1}^{+}, q}^{-\lambda}\left[\left(\zeta-\varsigma_{1}\right)^{\alpha} \prod_{j \neq \kappa}^{n} \Psi_{j}^{\delta j} \Psi_{\kappa}^{\eta}(\zeta)\right] \nabla_{\mathcal{C}_{1}^{+}, q}^{-\beta}\left[\prod_{j=1}^{n} \Psi_{j}^{\delta j}(\zeta)\right],
\end{aligned}
$$

gives the desired inequality (38).

Theorem 15. Let $\alpha>0, \eta \geq \delta_{\kappa}>0$ for any fixed $\kappa \in\{1,2,3$, $\cdots, n\}, \beta \in \mathbb{C}$ with $\Re(\beta)>0$, and $\hbar$ and $\Psi_{j}(j=1,2,3, \cdots, n)$ be the continuous positive decreasing functions defined on $\mathbb{T}_{t_{0}}$. Then, the time-fractional $q$-integral satisfies the inequality

$$
\frac{\nabla_{\mathcal{C}_{1}^{+}, q}^{-\beta}\left[\prod_{j \neq \kappa}^{n} \Psi_{j}^{\delta j} \Psi_{\kappa}^{\eta}(\zeta)\right] \nabla_{\mathcal{\zeta}_{1}^{+}, q}^{-\beta}\left[\hbar^{\alpha}(\zeta) \prod_{j=1}^{n} \Psi_{j}^{\delta j}(\zeta)\right]}{\nabla_{\mathcal{\zeta}_{1}^{\prime}, q}^{-\beta}\left[\hbar^{\alpha}(\zeta) \prod_{j \neq \kappa}^{n} \Psi_{j}^{\delta j} \Psi_{\kappa}^{\eta}(\zeta)\right] \nabla_{\mathcal{C}_{1}^{+}, q}^{-\beta}\left[\prod_{j=1}^{n} \Psi_{j}^{\delta j}(\zeta)\right]} \geq 1 .
$$

Proof. It follows from the given hypothesis that

$$
\left(\hbar^{\alpha}(\omega)-\hbar^{\alpha}(\varphi)\right)\left(\Psi_{\kappa}^{\eta-\delta \kappa}(\varphi)-\Psi_{\kappa}^{\eta-\delta \kappa}(\omega)\right) \geq 0,
$$

for any fixed $\kappa \in\{1,2,3, \cdots, n\}, \alpha>0, \eta \geq \delta_{\kappa}>0$, and $\varphi, \omega$ $\epsilon\left[\varsigma_{1}, \zeta\right]$.

Inequality (42) leads to

$$
\begin{aligned}
& \hbar^{\alpha}(\omega) \Psi_{\kappa}^{\eta-\delta \kappa}(\varphi)+\hbar^{\alpha}(\varphi) \Psi_{\kappa}^{\eta-\delta \kappa}(\omega)-\hbar^{\alpha}(\omega) \Psi_{\kappa}^{\eta-\delta \kappa}(\omega) \\
& -\hbar^{\alpha}(\varphi) \Psi_{\kappa}^{\eta-\delta \kappa}(\varphi) \geq 0 .
\end{aligned}
$$

Taking the product on both sides of (43) by $1 / \Gamma_{q}(\beta)$ $(\zeta-q \varphi)^{\beta-1 /} \prod_{j=1}^{n} \Psi_{j}^{\delta_{j}}(\varphi), \varphi \in\left(\varsigma_{1}, \zeta\right)$, and integrating for $\varphi$ over $\left(\varsigma_{1}, \zeta\right)$, we obtain

$$
\begin{aligned}
& \hbar^{\alpha}(\omega) \frac{1}{\Gamma_{q}(\beta)}(\zeta-q \varphi)^{\beta-1} \prod_{j=1}^{n} \Psi_{j}^{\delta_{j}}(\varphi) \Psi_{\kappa}^{\eta-\delta \kappa}(\varphi) \\
& +\hbar^{\alpha}(\varphi) \frac{1}{\Gamma_{q}(\beta)}(\zeta-q \varphi)^{\beta-1} \prod_{j=1}^{n} \Psi_{j}^{\delta_{j}}(\varphi) \Psi_{\kappa}^{\eta-\delta \kappa}(\omega) \\
& -\hbar^{\alpha}(\omega) \frac{1}{\Gamma_{q}(\beta)}(\zeta-q \varphi)^{\beta-1} \prod_{j=1}^{n} \Psi_{j}^{\delta_{j}}(\varphi) \Psi_{\kappa}^{\eta-\delta \kappa}(\omega) \\
& -\hbar^{\alpha}(\varphi) \frac{1}{\Gamma_{q}(\beta)}(\zeta-q \varphi)^{\beta-1} \prod_{j=1}^{n} \Psi_{j}^{\delta_{j}}(\varphi) \Psi_{\kappa}^{\eta-\delta \kappa}(\varphi) \geq 0 .
\end{aligned}
$$

Integrating (44) for $\varphi$ over $\left(\varsigma_{1}, \zeta\right)$, we have

$$
\begin{aligned}
& \hbar^{\alpha}(\omega) \frac{1}{\Gamma_{q}(\beta)} \int_{\zeta_{1}}^{\zeta}(\zeta-q \varphi)^{\beta-1} \prod_{j=1}^{n} \Psi_{j}^{\delta_{j}}(\varphi) \Psi_{\kappa}^{\eta-\delta \kappa}(\varphi) \nabla \varphi \\
& +\hbar^{\alpha}(\varphi) \frac{1}{\Gamma_{q}(\beta)} \int_{\zeta_{1}}^{\zeta}(\zeta-q \varphi)^{\beta-1} \prod_{j=1}^{n} \Psi_{j}^{\delta_{j}}(\varphi) \Psi_{\kappa}^{\eta-\delta \kappa}(\omega) \nabla \varphi \\
& -\hbar^{\alpha}(\omega) \frac{1}{\Gamma_{q}(\beta)} \int_{\zeta_{1}}^{\zeta}(\zeta-q \varphi)^{\beta-1} \prod_{j=1}^{n} \Psi_{j}^{\delta_{j}}(\varphi) \Psi_{\kappa}^{\eta-\delta \kappa}(\omega) \nabla \varphi \\
& -\hbar^{\alpha}(\varphi) \frac{1}{\Gamma_{q}(\beta)} \int_{\zeta_{1}}^{\zeta}(\zeta-q \varphi)^{\beta-1} \prod_{j=1}^{n} \Psi_{j}^{\delta_{j}}(\varphi) \Psi_{\kappa}^{\eta-\delta \kappa}(\varphi) \nabla \varphi \geq 0 .
\end{aligned}
$$

From (43), we clearly see that

$$
\begin{aligned}
& \hbar^{\alpha}(\omega) \nabla_{\zeta_{1}^{1}, q}^{-\beta}\left[\prod_{j \neq \kappa}^{n} \Psi_{j}^{\delta j} \Psi_{\kappa}^{\eta}(\zeta)\right]+\Psi_{\kappa}^{\eta-\delta \kappa}(\zeta) \nabla_{\zeta_{1}^{1}, q}^{-\beta}\left[\hbar^{\alpha}(\zeta) \prod_{j=1}^{n} \Psi_{j}^{\delta j}(\zeta)\right] \\
& -\hbar^{\alpha}(\omega) \Psi_{\kappa}^{\eta-\delta \kappa}(\omega) \nabla_{\epsilon_{1}^{1}, q}^{-\beta}\left[\prod_{j=1}^{n} \Psi_{j}^{\delta j}(\zeta)\right]-\nabla_{\zeta_{1}^{1}, q}^{-\beta}\left[\hbar^{\alpha}(\zeta) \prod_{j \neq \kappa}^{n} \Psi_{j}^{\delta j} \Psi_{\kappa}^{\eta}(\zeta)\right] \geq 0 .
\end{aligned}
$$

Again, taking the product on both sides of (46) by $1 /$ $\Gamma_{q}(\beta)(\zeta-q \theta) \prod_{j=1}^{n} \Psi_{j}^{\delta_{j}}(\omega), \omega \in\left(\varsigma_{1}, \zeta\right)$, and integrating for $\omega$ over $\left(\varsigma_{1}, \zeta\right)$, we have

$$
\begin{aligned}
\nabla_{\mathcal{C}_{1}^{+}, q}^{-\beta} & {\left[\prod_{j \neq \kappa}^{n} \Psi_{j}^{\delta j} \Psi_{\kappa}^{\eta}(\zeta)\right] \nabla_{\mathcal{C}_{1}^{+}, q}^{-\beta}\left[\hbar^{\alpha}(\zeta) \prod_{j=1}^{n} \Psi_{j}^{\delta j}(\zeta)\right] } \\
- & \nabla_{\mathcal{S}_{1}^{+}, q}^{-\beta}\left[\hbar^{\alpha}(\zeta) \prod_{j \neq \kappa}^{n} \Psi_{j}^{\delta j} \Psi_{\kappa}^{\eta}(\zeta)\right] \nabla_{\mathcal{C}_{1}^{+}, q}^{-\beta}\left[\prod_{j=1}^{n} \Psi_{j}^{\delta j}(\zeta)\right] \geq 0,
\end{aligned}
$$

which completes the proof of the desired inequality (41).

Theorem 16. Let $\alpha>0, \eta \geq \delta_{\kappa}>0$ for any fixed $\kappa \in\{1,2,3$, $\cdots, n\}, \beta, \lambda \in \mathbb{C}$, with $\mathfrak{R}(\beta)>0, \mathfrak{R}(\lambda)>0,\left\{\Psi_{j}, j=1,2,3, \cdots\right.$, $n\}$ be a sequence of continuous positive decreasing functions defined on $\mathbb{T}_{t_{0}}$ and $\hbar$ be a continuous positive increasing functions defined on $\mathbb{T}_{t_{0}}$. Then 


$$
\begin{aligned}
& \left(\nabla_{\mathcal{C}_{1}^{+}, q}^{-\beta}\left[\prod_{j \neq \kappa}^{n} \Psi_{j}^{\delta j} \Psi_{\kappa}^{\eta}(\zeta)\right] \nabla_{\mathcal{C}_{1}^{+}, q}^{-\lambda}\left[\hbar^{\alpha}(\zeta) \prod_{j=1}^{n} \Psi_{j}^{\delta j}(\zeta)\right]\right. \\
& \left.+\nabla_{\mathcal{C}_{1}^{+}, q}^{-\beta}\left[\hbar^{\alpha}(\zeta) \prod_{j=1}^{n} \Psi_{j}^{\delta j}(\zeta)\right] \nabla_{\zeta_{1}^{+}, q}^{-\lambda}\left[\prod_{j \neq \kappa}^{n} \Psi_{j}^{\delta j} \Psi_{\kappa}^{\eta}(\zeta)\right]\right) / \\
& \quad\left(\nabla_{\mathcal{C}_{1}^{+}, q}^{-\lambda}\left[\prod_{j=1}^{n} \Psi_{j}^{\delta j}(\zeta)\right] \nabla_{\mathcal{C}_{1}^{+}, q}^{-\beta}\left[\hbar^{\alpha}(\zeta) \prod_{j \neq \kappa}^{n} \Psi_{j}^{\delta j} \Psi_{\kappa}^{\eta}(\zeta)\right]\right. \\
& \left.+\nabla_{\mathcal{C}_{1}^{+}, q}^{-\lambda}\left[\hbar^{\alpha}(\zeta) \prod_{j \neq \kappa}^{n} \Psi_{j}^{\delta j} \Psi_{\kappa}^{\eta}(\zeta)\right] \nabla_{\mathcal{C}_{1}^{+}, q}^{-\beta}\left[\prod_{j=1}^{n} \Psi_{j}^{\delta j}(\zeta)\right]\right) \geq 1 .
\end{aligned}
$$

Proof. Multiplying both sides of (46) by $1 / \Gamma_{q}(\lambda)(\zeta-q \omega)^{\lambda-1 /}$ $\prod_{j=1}^{n} \Psi_{j}^{\delta_{j}}(\omega), \omega \in\left(\varsigma_{1}, \zeta\right)$, and integrating for $\omega$ over $\left(\varsigma_{1}, \zeta\right)$, we have

$$
\begin{aligned}
\nabla_{\zeta_{1}^{+}, q}^{-\beta} & {\left[\prod_{j \neq \kappa}^{n} \Psi_{j}^{\delta j} \Psi_{\kappa}^{\eta}(\zeta)\right] \nabla_{\zeta_{1}^{+}, q}^{-\lambda}\left[\hbar^{\alpha}(\zeta) \prod_{j=1}^{n} \Psi_{j}^{\delta j}(\zeta)\right] } \\
+ & \nabla_{\mathcal{C}_{1}^{+}, q}^{-\beta}\left[\hbar^{\alpha}(\zeta) \prod_{j=1}^{n} \Psi_{j}^{\delta j}(\zeta)\right] \nabla_{\mathcal{C}_{1}^{+}, q}^{-\lambda}\left[\prod_{j \neq \kappa}^{n} \Psi_{j}^{\delta j} \Psi_{\kappa}^{\eta}(\zeta)\right] \\
- & \nabla_{\mathcal{C}_{1}^{+}, q}^{-\lambda}\left[\prod_{j=1}^{n} \Psi_{j}^{\delta j}(\zeta)\right] \nabla_{\mathcal{C}_{1}^{+}, q}^{-\beta}\left[\hbar^{\alpha}(\zeta) \prod_{j \neq \kappa}^{n} \Psi_{j}^{\delta j} \Psi_{\kappa}^{\eta}(\zeta)\right] \\
- & \nabla_{\mathcal{C}_{1}^{\prime}, q}^{-\lambda}\left[\hbar^{\alpha}(\zeta) \prod_{j \neq \kappa}^{n} \Psi_{j}^{\delta j} \Psi_{\kappa}^{\eta}(\zeta)\right] \nabla_{\mathcal{C}_{1}^{+}, q}^{-\beta}\left[\prod_{j=1}^{n} \Psi_{j}^{\delta j}(\zeta)\right] \geq 0 .
\end{aligned}
$$

It follows that

$$
\begin{aligned}
\nabla_{\mathcal{C}_{1}^{+}, q}^{-\beta} & {\left[\prod_{j \neq \kappa}^{n} \Psi_{j}^{\delta j} \Psi_{\kappa}^{\eta}(\zeta)\right] \nabla_{\mathcal{C}_{1}^{+}, q}^{-\lambda}\left[\hbar^{\alpha}(\zeta) \prod_{j=1}^{n} \Psi_{j}^{\delta j}(\zeta)\right] } \\
+ & \nabla_{\mathcal{C}_{1}^{+}, q}^{-\beta}\left[\hbar^{\alpha}(\zeta) \prod_{j=1}^{n} \Psi_{j}^{\delta j}(\zeta)\right] \nabla_{\mathcal{C}_{1}^{+}, q}^{-\lambda}\left[\prod_{j \neq \kappa}^{n} \Psi_{j}^{\delta j} \Psi_{\kappa}^{\eta}(\zeta)\right] \\
\geq & \nabla_{\mathcal{C}_{1}^{+}, q}^{-\lambda}\left[\prod_{j=1}^{n} \Psi_{j}^{\delta j}(\zeta)\right] \nabla_{\mathcal{C}_{1}^{+}, q}^{-\beta}\left[\hbar^{\alpha}(\zeta) \prod_{j \neq \kappa}^{n} \Psi_{j}^{\delta j} \Psi_{\kappa}^{\eta}(\zeta)\right] \\
+ & \nabla_{\mathcal{C}_{1}^{+}, q}^{-\lambda}\left[\hbar^{\alpha}(\zeta) \prod_{j \neq \kappa}^{n} \Psi_{j}^{\delta j} \Psi_{\kappa}^{\eta}(\zeta)\right] \nabla_{\mathcal{C}_{1}^{+}, q}^{-\beta}\left[\prod_{j=1}^{n} \Psi_{j}^{\delta j}(\zeta)\right]
\end{aligned}
$$

Dividing both sides of the above inequality by

$$
\begin{aligned}
& \nabla_{\zeta_{1}^{+}, q}^{-\lambda} {\left[\prod_{j=1}^{n} \Psi_{j}^{\delta j}(\zeta)\right] \nabla_{\zeta_{1}^{+}, q}^{-\beta}\left[\hbar^{\alpha}(\zeta) \prod_{j \neq \kappa}^{n} \Psi_{j}^{\delta j} \Psi_{\kappa}^{\eta}(\zeta)\right] } \\
&+\nabla_{\mathcal{C}_{1}^{+}, q}^{-\lambda}\left[\hbar^{\alpha}(\zeta) \prod_{j \neq \kappa}^{n} \Psi_{j}^{\delta j} \Psi_{\kappa}^{\eta}(\zeta)\right] \nabla_{\mathcal{C}_{1}^{+}, q}^{-\beta}\left[\prod_{j=1}^{n} \Psi_{j}^{\delta j}(\zeta)\right],
\end{aligned}
$$

gives desired inequality (48).

\section{Conclusion}

In this note, we have derived certain variants by using the time-fractional $q$-calculus operator related to a class of $n$ positive continuous, and decreasing functions on the interval $\left[\varsigma_{1}, \varsigma_{2}\right]$ are elaborated. In [66], Liu et al. investigated thoughtprovoking integral inequalities for continuous functions on $\left[\varsigma_{1}, \varsigma_{2}\right]$. Recently, Dahmani [67] has presented the more generalizations of the work of [66] by utilizing the RiemannLiouville fractional integral operators. If we take into account $\mathbb{T}=\mathbb{R}$ and $q=1$, then our findings are the special cases of the results proposed by Dahmani [67]. The established relationship highlighted the importance of selecting appropriate combinations and validated $q$-fractional time scale approaches for special relativity theory and quantum mechanics. From the existence and uniqueness viewpoint, it is found that the $q$-fractional order controls potentially provide the tools to better represent measured that cannot be fit to the classical model.

\section{Data Availability}

No data were used to support this study.

\section{Conflicts of Interest}

The authors declare that there are no conflicts of interest regarding the publication of this paper.

\section{Authors' Contributions}

L. Xu provided the main ideas of the article and carried out the proof of Theorem 9. Y.-M. Chu drafted the manuscript and carried out the proof of Theorem 10. S. Rashid carried out the proof of Theorem 11 and Theorem 12, completed the final revision, and submitted the article. A. A.El-Deeb carried out the proof of Theorems 13 and 14. K. S. Nisar carried out the proof of Theorems 15 and 16 . All authors read and approved the final manuscript.

\section{Acknowledgments}

The authors would like to express their sincere thanks to the editor and the anonymous reviewers for their helpful comments and suggestions. This work was supported by the Natural Science Foundation of China (Grant Nos. 61673169, $11971142,11871202,11301127,11701176,11626101$, and 11601485).

\section{References}

[1] Z. Dai and F. Wen, "Another improved Wei-Yao-Liu nonlinear conjugate gradient method with sufficient descent property," Applied Mathematics and Computation, vol. 218, no. 14, pp. 7421-7430, 2012.

[2] Y. Jiang and J. Ma, "Spectral collocation methods for Volterra-integro differential equations with noncompact kernels," Journal of Computational and Applied Mathematics, vol. 244, pp. 115-124, 2013. 
[3] Z.-F. Dai and F.-H. Wen, "Robust CVaR-based portfolio optimization under a genal affine data perturbation uncertainty set," Journal of Computational Analysis and Applications, vol. 16, no. 1, pp. 93-103, 2014.

[4] C. Huang, S. Guo, and L. Liu, "Boundedness on Morrey space for Toeplitz type operator associated to singular integral operator with variable Calderón-Zygmund kernel," Journal of Mathematical Inequalities, vol. 8, no. 3, pp. 453-464, 2014.

[5] W. Zhou and F. Wang, "A PRP-based residual method for large-scale monotone nonlinear equations," Applied Mathematics and Computation, vol. 261, pp. 1-7, 2015.

[6] X.-P. Fang, Y.-J. Deng, and J. Li, "Plasmon resonance and heat generation in nanostructures," Mathematicsl Methods in the Applied Sciences, vol. 38, no. 18, pp. 4663-4672, 2016.

[7] J.-L. Li, G.-Y. Sun, and R.-M. Zhang, “The numerical solution of scattering by infinite rough interfaces based on the integral equation method," Computers \& Mathematcs with Applications, vol. 71, no. 7, pp. 1491-1502, 2016.

[8] L. Duan and C.-X. Huang, "Existence and global attractivity of almost periodic solutions for a delayed differential neoclassical growth model," Mathematicsl Methods in the Applied Sciences, vol. 40, no. 3, pp. 814-822, 2017.

[9] W. Wang, "On $A$-stable one-leg methods for solving nonlinear Volterra functional differential equations," Applied Mathematics and Computation, vol. 314, pp. 380-390, 2017.

[10] Y.-X. Tan, C.-X. Huang, B. Sun, and T. Wang, "Dynamics of a class of delayed reaction-diffusion systems with Neumann boundary condition," Journal of Mathematical Analysis and Applications, vol. 458, no. 2, pp. 1115-1130, 2018.

[11] J.-F. Wang, X.-Y. Chen, and L.-H. Huang, "The number and stability of limit cycles for planar piecewise linear systems of node-saddle type," Journal of Mathematical Analysis and Applications, vol. 469, no. 1, pp. 405-427, 2019.

[12] C.-X. Huang, H. Zhang, and L.-H. Huang, "Almost periodicity analysis for a delayed Nicholson's blowflies model with nonlinear density-dependent mortality term," Communications on Pure \& Applied Analysis, vol. 18, no. 6, pp. 33373349, 2019.

[13] W.-J. Zhou, "On the convergence of the modified LevenbergMarquardt method with a nonmonotone second order Armijo type line search," Journal of Computational and Applied Mathematics, vol. 239, pp. 152-161, 2013.

[14] W.-J. Zhou and X.-L. Chen, "On the convergence of a modified regularized Newton method for convex optimization with singular solutions," Journal of Computational and Applied Mathematics, vol. 239, pp. 179-188, 2013.

[15] L. Zhang and S.-Y. Jian, "Further studies on the Wei-Yao-Liu nonlinear conjugate gradient method," Applied Mathematics and Computation, vol. 219, no. 14, pp. 7616-7621, 2013.

[16] T. Abdeljawad and D. Baleanu, "Monotonicity results for fractional difference operators with discrete exponential kernels," Advances in Difference Equations, vol. 2017, 2017.

[17] T. Abdeljawad and D. Baleanu, "On fractional derivatives with exponential kernel and their discrete versions," Reports on Mathematical Physics, vol. 80, no. 1, pp. 11-27, 2017.

[18] A. A. Kilbas, H. M. Srivastava, and J. J. Trujillo, Theory and Applications of Fractional Differential Equations, Elsevier Science B.V, Amsterdam, 2006.

[19] R. Khalil, M. Al Horani, A. Yousef, and M. Sababheh, "A new definition of fractional derivative," Journal of Computational and Applied Mathematics, vol. 264, pp. 65-70, 2014.
[20] K. S. Miller and B. Ross, An Introduction to the Fractional Calculus and Fractional Differential Equations, John Wiley \& Sons, New York, 1993.

[21] I. Podlubny, Fractional Differential Equations, Academic Press, San Diego, 1999.

[22] S. G. Samko, A. A. Kilbas, and O. I. Marichev, Fractional Integrals and Derivatives, Gordon and Breach Science Publishers, Yverdon, 1993.

[23] S. Hilger, Ein Mabkettenkalkül Mit Anwendung Auf Zentrumsmannigfaltigkeiten, [Ph.D thesis], Universität Würzburg, 1988.

[24] S. Hilger, "Differential and difference calculus-unified!," Nonlinear Analysis, vol. 30, no. 5, pp. 2683-2694, 1997.

[25] S. Hilger, "Analysis on measure chains-a unified approach to continuous and discrete calculus," Results in Mathematics, vol. 18, no. 1-2, pp. 18-56, 1990.

[26] M. Bohner and A. Peterson, Dynamic Equations on Time Scales, Birkhäuser Boston, Boston, 2001.

[27] C. Dinu, "Hermite-Hadamard inequality on time scales," Journal of Inequalities and Applications, vol. 2008, Article ID 287947, 2008.

[28] C. Dinu, "Ostrowski type inequalities on time scales," Annals of the University of Craiova-Mathematics and Computer Science Series, vol. 34, pp. 43-58, 2007.

[29] N. R. O. Bastos, D. Mozyrska, and D. F. M. Torres, "Fractional derivatives and integrals on time scales via the inverse generalized Laplace transform," International Journal of Computational Mathematics, vol. 11, 9 pages, 2011.

[30] R. P. Agarwal, "Certain fractionalq-integrals andq-derivatives," Proceedings of the Cambridge Philosophical Society, vol. 66, no. 2, pp. 365-370, 1969.

[31] W. A. Al-Salam and A. Verma, "A fractional Leibniz $q$-formula," Pacific Journal of Mathematics, vol. 60, no. 2, pp. 1-9, 1975.

[32] G. E. Andrews, R. Askey, and R. Roy, Special Functions, Cambridge University Press, Cambridge, 2013.

[33] F. M. Atici and P. W. Eloe, "A transform method in discrete fractional calculus," International Journal of Difference Equations, vol. 2, no. 2, pp. 165-176, 2007.

[34] M. A. Latif, S. Rashid, S. S. Dragomir, and Y.-M. Chu, "Hermite-Hadamard type inequalities for co-ordinated convex and qausi-convex functions and their applications," Journal of Inequalities and Applications, vol. 2019, no. 1, 2019.

[35] M. U. Awan, N. Akhtar, S. Iftikhar, M. A. Noor, and Y.-M. Chu, "New Hermite-Hadamard type inequalities for $n$-polynomial harmonically convex functions," Journal of Inequalities and Applications, vol. 2020, 2020.

[36] M. Adil Khan, N. Mohammad, E. R. Nwaeze, and Y.-M. Chu, "Quantum Hermite-Hadamard inequality by means of a Green function," Advances in Difference Equations, vol. 2020, 2020.

[37] A. Iqbal, M. Adil Khan, S. Ullah, and Y.-M. Chu, "Some new Hermite-Hadamard-type inequalities associated with conformable fractional integrals and their applications," Journal of Function Spaces, vol. 2020, Article ID 9845407, 18 pages, 2020.

[38] M. U. Awan, S. Talib, Y.-M. Chu, M. A. Noor, and K. I. Noor, "Some new refinements of Hermite-Hadamard-type inequalities involving $\Psi_{k}$-Riemann-Liouville fractional integrals and applications," Mathematical Problems in Engineering, vol. 2020, Article ID 3051920, 10 pages, 2020. 
[39] I. Abbas Baloch and Y.-M. Chu, "Petrović-type inequalities for harmonic $h$-convex functions," Journal of Function Spaces, vol. 2020, Article ID 3075390, 7 pages, 2020.

[40] S. Rashid, F. Jarad, H. Kalsoom, and Y.-M. Chu, "On PólyaSzegö and $\mathrm{C}^{\prime}$ ebyšev type inequalities via generalized $k$-fractional integrals," Advances in Difference Equations, vol. 2020, 2020.

[41] S. Rashid, M. A. Noor, K. I. Noor, and Y.-M. Chu, "Ostrowski type inequalities in the sense of generalized $\mathscr{K}$-fractional integral operator for exponentially convex functions," AIMS Mathematics, vol. 5, no. 3, pp. 2629-2645, 2020.

[42] S. Rashid, F. Jarad, and Y.-M. Chu, "A note on reverse Minkowski inequality via generalized proportional fractional integral operator with respect to another function," Mathematical Problems in Engineering, vol. 2020, Article ID 7630260, 12 pages, 2020.

[43] S. Khan, M. Adil Khan, and Y.-M. Chu, "Converses of the Jensen inequality derived from the Green functions with applications in information theory," Mathematical Methods in the Applied Sciences, vol. 43, pp. 2577-2587, 2020.

[44] M. A. Khan, J. Pečarić, and Y.-M. Chu, "Refinements of Jensen's and McShane's inequalities with applications," AIMS Mathematics, vol. 5, no. 5, pp. 4931-4945, 2020.

[45] T.-H. Zhao, L. Shi, and Y.-M. Chu, "Convexity and concavity of the modified Bessel functions of the first kind with respect to Hölder means," Revista de la Real Academia de Ciencias Exactas, Físicas y Naturales. Serie A. Matemáticas, vol. 114, no. 2, 2020.

[46] M.-K. Wang, M.-Y. Hong, Y.-F. Xu, Z.-H. Shen, and Y.$\mathrm{M}$. Chu, "Inequalities for generalized trigonometric and hyperbolic functions with one parameter," Journal of Mathematical Inequalities, vol. 14, no. 1, pp. 1-21, 2020.

[47] Y.-M. Chu, M. Adil Khan, T. Ali, and S. S. Dragomir, "Inequalities for $\alpha$-fractional differentiable functions," Journal of Inequalities and Applications, vol. 2017, no. 1, 2017.

[48] S.-S. Zhou, S. Rashid, F. Jarad, H. Kalsoom, and Y.-M. Chu, "New estimates considering the generalized proportional Hadamard fractional integral operators," Advances in Difference Equations, vol. 2020, no. 1, 2020.

[49] Y. Khurshid, M. Adil Khan, and Y.-M. Chu, "Conformable fractional integral inequalities for $G G$ - and $G A$-convex functions," AIMS Mathematics, vol. 5, no. 5, pp. 5012-5030, 2020.

[50] S. Rafeeq, H. Kalsoom, S. Hussain, S. Rashid, and Y.-M. Chu, "Delay dynamic double integral inequalities on time scales with applications," Advances in Difference Equations, vol. 2020, no. 1, 2020.

[51] S. Rashid, İ. İşcan, D. Baleanu, and Y.-M. Chu, "Generation of new fractional inequalities via n polynomials s-type convexity with applications," Advances in Difference Equations, vol. 2020, no. 1, 2020.

[52] M.-K. Wang, H.-H. Chu, and Y.-M. Chu, "Precise bounds for the weighted Hölder mean of the complete $p$-elliptic integrals," Journal of Mathematical Analysis and Applications, vol. 480, no. 2, Article ID 123388, 2019.

[53] M.-K. Wang, Z.-Y. He, and Y.-M. Chu, "Sharp power mean inequalities for the generalized elliptic integral of the first kind," Computational Methods and Function Theory, vol. 20, no. 1, pp. 111-124, 2020.

[54] T.-H. Zhao, M.-K. Wang, and Y.-M. Chu, "A sharp double inequality involving generalized complete elliptic integral of the first kind," AIMS Mathematics, vol. 5, no. 5, pp. 4512 $4528,2020$.

[55] M.-K. Wang, H.-H. Chu, Y.-M. Li, and Y.-M. Chu, “Answers to three conjectures on convexity of three functions involving complete elliptic integrals of the first kind," Applicable Analysis and Discrete Mathematics, vol. 14, pp. 255-271, 2020.

[56] W.-M. Qian, Z.-Y. He, and Y.-M. Chu, "Approximation for the complete elliptic integral of the first kind," Revista de la Real Academia de Ciencias Exactas, Físicas y Naturales. Serie A. Matemáticas, vol. 114, no. 2, 2020.

[57] Z.-H. Yang, W.-M. Qian, W. Zhang, and Y.-M. Chu, "Notes on the complete elliptic integral of the first kind," Mathematical Inequalities \& Applications, vol. 23, no. 1, pp. 77-93, 2020.

[58] S. Zaheer Ullah, M. Adil Khan, and Y.-M. Chu, "A note on generalized convex functions," Journal of Inequalities and Applications, vol. 2019, no. 1, 2019.

[59] M. U. Awan, N. Akhtar, A. Kashuri, M. A. Noor, and Y.-M. Chu, " $2 D$ approximately reciprocal $\rho$-convex functions and associated integral inequalities," AIMS Mathematics, vol. 5, no. 5, pp. 4662-4680, 2020.

[60] S. Rashid, R. Ashraf, M. A. Noor, K. I. Noor, and Y.-M. Chu, "New weighted generalizations for differentiable exponentially convex mapping with application," AIMS Mathematics, vol. 5, no. 4, pp. 3525-3546, 2020.

[61] W.-M. Qian, Y.-Y. Yang, H.-W. Zhang, and Y.-M. Chu, "Optimal two-parameter geometric and arithmetic mean bounds for the Sándor-Yang mean," Journal of Inequalities and Applications, vol. 2019, 2019.

[62] W.-M. Qian, Z.-Y. He, H.-W. Zhang, and Y.-M. Chu, "Sharp bounds for Neuman means in terms of two-parameter contraharmonic and arithmetic mean," Journal of Inequalities and Applications, vol. 2019, 2019.

[63] B. Wang, C.-L. Luo, S.-H. Li, and Y.-M. Chu, "Sharp oneparameter geometric and quadratic means bounds for the Sándor-Yang means," Revista de la Real Academia de Ciencias Exactas, Físicas y Naturales. Serie A. Matemáticas, vol. 114, no. $1,2020$.

[64] H. Khan, T. Abdeljawad, C. Tunç, A. Alkhazzan, and A. Khan, "Minkowski's inequality for the AB-fractional integral operator," Journal of Inequalities and Applications, vol. 2019, no. 1, 2019.

[65] H. Khan, C. Tunç, D. Baleanu, A. Khan, and A. Alkhazzan, "Inequalities for n-class of functions using the Saigo fractional integral operator," Revista de la Real Academia de Ciencias Exactas, Físicas y Naturales. Serie A. Matemáticas, vol. 113, no. 3, pp. 2407-2420, 2019.

[66] W.-J. Liu, Q. A. Ngô, and V. N. Huy, "Several interesting integral inequalities," Journal of Mathematical Inequalities, vol. 3, no. 2, pp. 201-212, 2009.

[67] Z. Dahmani, "New classes of integral inequalities of fractional order," Matematiche, vol. 69, no. 1, pp. 237-247, 2014. 